\title{
Ein finites Stab-Aktor-Element und seine Anwendung in der Optimierung adaptiver Tragwerke
}

\author{
Jan Gade', Florian Geiger ${ }^{1}$, Malte von Scheven ${ }^{1}$, Manfred Bischoff ${ }^{1}$ \\ ${ }^{1}$ Universität Stuttgart, Institut für Baustatik und Baudynamik, Pfaffenwaldring 7, \\ 70569 Stuttgart
}

In der Simulation von adaptiven Tragwerken wird die Aktuierung häufig durch Einführung zusätzlicher Lastfälle oder Randbedingungen modelliert. Insbesondere bei der Berücksichtigung geometrisch nichtlinearer Effekte bedeutet dieses Vorgehen einen relativ hohen Modellierungsaufwand. Wir stellen ein finites Stab-Aktor-Element zur statischen und transienten Simulation von adaptiven Fachwerkstrukturen vor, das die Aktorwirkung beinhaltet und so den Modellierungsaufwand verringert sowie potentielle Fehlerquellen vermeidet. Das Element wird aus einem beschränkten Variationsprinzip hergeleitet.

Ein weiterer Aspekt ist das Konzept der Redundanzmatrizen [1], mit dem auf Basis der strukturmechanischen Einsicht in das Tragverhalten Schlussfolgerungen in Hinblick auf die Adaptierbarkeit eines Tragwerks oder einzelner Tragwerksteile möglich ist. Beispiele einfacher Fachwerkstrukturen unter Einsatz des vorgestellten Elements werden gezeigt. Hierbei wird auf den prinzipiellen Unterschied zwischen der nachträglichen Adaption eines zuvor optimierten passiven Tragwerks und der Optimierung eines adaptiven Tragwerks eingegangen.

\section{Literatur}

Ströbel, D.: Die Anwendung der Ausgleichungsrechnung auf elastomechanische Syste- me. Dissertation, Institut für Anwendungen der Geodäsie im Bauwesen, Universität Stuttgart, 1995 